\title{
Alcoolismo Parental e suas RePercussões Sobre Crianças e Adolescentes: uma REVISÃO BIBLIOGRÁFICA.
}

Daniela Viganó Zanoti-Jeronymo ${ }^{1}$; Ana Maria Pimenta Carvalho ${ }^{2}$

\section{Resumo}

Um dos fatores de risco para o alcoolismo na vida adulta é ser filho de alcoolista. Com esse tema em foco, o presente trabalho objetivou revisar a literatura dos últimos 5 anos nas bases de dados Medline, Psyclit e Lilacs, que abordavam crianças e ou adolescentes filhos de alcoolistas. De 300 pesquisas, foram selecionados 27 trabalhos. A análise dos trabalhos resultou em 3 áreas temáticas: alcoolismo parental associado com características afetivas e comportamentais da criança ou do adolescente; em co-ocorrência com outro distúrbio e características da criança ou do adolescente; e fatores de proteção à saúde mental da criança ou do adolescente.

Palavras-chave: alcoholismo, relações familiares

\section{ALCOHOLISMO PARENTAL Y SUS REPERCUSIONES SOBRE NIÑOS Y ADOLESCENTES: UNA REVISIÓN BIBLIOGRÁFICA.}

Resumen

Uno de los factores de riesgo para el alcoholismo en la vida adulta es ser hijo de alcohólico. Enfocando ese tema, la finalidad de este trabajo fue revisar la literatura de los últimos 5 años en las bases de datos Medline, Psyclit y Lilacs, sobre niños y/o adolescentes hijos de alcohólicos. De 300 investigaciones, se seleccionaron 27 trabajos. El análisis de los trabajos resultó en 3 áreas temáticas: tener padres alcohólicos asociado con características afectivas y comportamentales del niño o del adolescente; en co-ocurrencia con otro disturbio y características del niño o del adolescente; y factores de protección a la salud mental del niño o del adolescente.

Palabras clave: alcoholismo, relaciones familiares

\section{PARENTAL ALCOHOLISM AND CONSECUENCIS ON CHILDREN AND ADOLESCENTES: A}

\section{REVIEW.}

\section{Abstract}

One risk factor for developing alcohol addiction in adult life is being the child of an alcoholic. In this context, we present a review of literature published in the last 5 years in Medline, Psyclit and Lilacs on children and adolescents with alcohol-addicted parents. Out of 300 studies, 27 papers were selected, resulting in 3 theme areas: having alcohol-addicted parents associated with the child's or adolescent's affective and behavioral characteristics; cooccurrence with another disorder and the child's or adolescent's characteristics; and factors to protect the child's or adolescent's mental health.

Key words: alcoholism, family relations

\footnotetext{
${ }^{1}$ Enfermeira, Mestre em Enfermagem Psiquiátrica, Professora da Universidade Estadual do Centro Oeste - UNICENTRO, $e$ mail: danielazanoti@bol.com.br

${ }^{2}$ Psicóloga, Doutora em Psicologia, Professora da Escola de Enfermagem de Ribeirão Preto - EERP/USP.
} 


\section{INTRODUÇÃO}

O consumo de álcool sofreu, nos últimos trinta anos, aumento significativo e, segundo estudos da OMS, aparece associado ao crescimento da indústria de bebidas alcoólicas, ao desenvolvimento do turismo em massa e ao desemprego ${ }^{(1)}$. O consumo de bebida alcoólica é muito difundido no Brasil. Cerca de 84\% da população brasileira faz uso ocasional de bebidas alcoólicas e os índices de dependência variam de 3 a $15 \%{ }^{2-3)}$.

Grant $^{(4)}$, a partir de uma pesquisa epidemiológica, encontrou que, para crianças e adolescentes menores de dezoito anos, uma em cada quatro está exposta ao abuso do álcool no ambiente familiar.

Nos últimos anos houve grande proliferação de pesquisas investigando o campo do alcoolismo em geral, proporcionando novo entendimento de que o desenvolvimento (etiologia) do alcoolismo resulta da influência de múltiplos fatores relacionados com o uso de álcool, bem como sua progressão e intensidade do beber ${ }^{(5)}$.

Nessa perspectiva, dentro dos múltiplos fatores - biológico, psicológico e social, as influências originadas no ambiente familiar, principalmente influências parentais, são relevantes para o desenvolvimento do abuso e dependência de álcool em seus descendentes ${ }^{(6)}$.

Vários pesquisadores têm focalizado filhos de alcoolistas pelo seu elevado risco de experienciar eventos negativos em seu ambiente familiar durante seu desenvolvimento ${ }^{(7-10)}$. O ambiente familiar e as maneiras como os membros interagem podem contribuir para o risco dos problemas observados em filhos de alcoolistas ${ }^{(11)}$.

Em geral, o alcoolismo parental é associado à variedade de resultados negativos para as crianças e os adolescentes. Os resultados de alguns estudos demonstram que filhos de alcoolistas exibem elevadas taxas de psicopatologias, sendo que esses são aproximadamente quatro a seis vezes mais prováveis, do que a população geral, de desenvolver problemas com abuso de álcool ${ }^{(12-14)}$. Além disso, ansiedade, depressão e desordem de comportamento

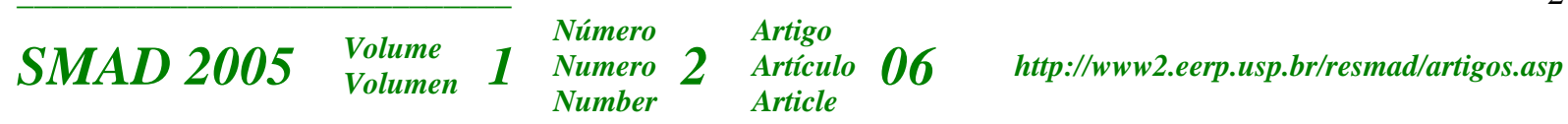


externalizados e internalizados são mais comuns em filhos de alcoolistas do que em filhos de não-alcoolistas ${ }^{(15-16)}$.

Os achados dessas análises têm levado à ressonância de um claro consenso da interligação entre ser filho de alcoolista, apresentar alguns resultados negativos durante o seu desenvolvimento e possível prognóstico de problemas com uso de álcool.

Diante do exposto, este artigo pretende revisar pesquisas que abordam o tema 'filhos de alcoolistas’ dentro de uma perspectiva teórica da concepção ecológica do desenvolvimento $^{(17)}$, buscando identificar condições de vulnerabilidade e de proteção à criança e/ou ao adolescente expostos ao alcoolismo familiar.

\section{METODOLOGIA}

O objeto do presente trabalho foi revisar pesquisas científicas que abordassem o tema filhos de alcoolistas, contidas em periódicos indexados nacionais e internacionais, para tanto o trabalho dividiu-se em duas etapas. Primeiramente realizou-se uma pesquisa bibliográfica nas bases de dados Psyclit, Medline e Lilacs utilizando, para isto, as palavras-chave alcoolismo e filhos. A delimitação do período de busca foi do ano de 1995 a 2000. Nessa primeira etapa a intenção foi de identificar trabalhos que abordassem o tema em questão. Foram encontrados um total de 300 relatos de pesquisas. Na segunda etapa, realizou-se a análise e distribuição das pesquisas por área temática e posteriormente foram selecionadas as pesquisas que atendessem os objetivos propostos e que abordassem apenas crianças e adolescentes filhos de alcoolistas. Utilizando esses critérios de exclusão, do total de 300 trabalhos, chegou-se à seleção de 27 artigos.

\section{RESULTADO E DISCUSSÃO}

$\begin{array}{llll}\text { SMAD } 2005 & \begin{array}{l}\text { Volume } \\ \text { Volumen }\end{array} & \begin{array}{l}\text { Número } \\ \text { Numero } \\ \text { Number }\end{array} & \begin{array}{l}\text { Artigo } \\ \text { Artículo } 06 \\ \text { Article }\end{array}\end{array}$ http://www2.eerp.usp.br/resmad/artigos.asp


Os resultados serão apresentados enfocando as principais áreas temáticas dos estudos que abordaram especificamente crianças e adolescentes filhos de alcoolistas. Entende-se, no presente trabalho, como área temática, o foco principal dos estudos.

Após a revisão detalhada do conteúdo desses artigos foram selecionados para a segunda etapa do trabalho, o total de 27 artigos. Esses foram divididos em três categorias distintas com agrupamento de temática em comum, para apresentação dos resultados.

\section{1) Alcoolismo parental e características afetivas e comportamentais da criança ou}

\section{do adolescente}

- Quanto a problemas comportamentais/afetivos

Crianças em idade escolar, filhos de pais alcoolistas, freqüentemente apresentam problemas acadêmicos e de comportamento quando comparadas a crianças e adolescentes filhos de não-alcoolistas. Focalizando o desempenho acadêmico de filhos de alcoolistas, constatou-se que esses, comparativamente a filhos de não-alcoolistas, apresentaram notas mais baixas e exibiam em relação às tarefas escolares, especialmente matemática, fraca organização e motivação ${ }^{(18)}$.

Em estudo realizado em uma missão indígena nos EUA, verificou-se, em crianças filhas de alcoolistas, escores significantemente maiores de problemas de comportamento internalizados e externalizados - quando comparados aos filhos de não-alcoolistas, e esses resultados negativos foram mais acentuados para os meninos ${ }^{(19)}$.

Focalizando as psicopatologias, realizações acadêmicas e o risco para desenvolver alcoolismo, em filhos de alcoolistas e filhos de não-alcoolistas, com idade de 8 a 18 anos, conclui-se que crianças que tinham alcoolismo multigeracional em suas famílias apresentavam maior risco para o desenvolvimento de psicopatologias ${ }^{(20)}$.

O que esses estudos sugerem é que parece haver interligação entre o alcoolismo parental, maiores índices de problemas de comportamento e desordens afetivas e baixo

\begin{tabular}{llll}
\hline SMAD 2005 & $\begin{array}{l}\text { Volume } \\
\text { Volumen } 1\end{array}$ & $\begin{array}{l}\text { Número } \\
\text { Numero } \\
\text { Number }\end{array}$ & $\begin{array}{l}\text { Artigo } \\
\text { Artículo } \\
\text { Article }\end{array}$
\end{tabular}$\quad$ http://www2.eerp.usp.br/resmad/artigos.asp


rendimento escolar. Essas observações podem ser consideradas indicadores para o diagnóstico de desenvolvimento de psicopatologias, inclusive alcoolismo.

Comparando filhos de alcoolistas com filhos de não-alcoolistas, quanto à vergonha, auto-estima, suporte para o aprendizado e relacionamento com o professor, não foram encontradas diferenças na mensuração do suporte social e vergonha, mas sim, em relação à auto-estima e relacionamento com os professores. Filhos de alcoolistas apresentaram resultados significantemente inferiores quando comparados com filhos de não-alcoolistas. Meninos filhos de alcoolistas demonstraram maiores índices de problemas de comportamento e baixa auto-estima comparativamente a meninas filhas de alcoolistas ${ }^{(21)}$.

Resultados semelhantes para meninos filhos de alcoolistas foram encontrados quando foram examinadas a associação entre alcoolismo parental e ausência parental no desenvolvimento e estabilidade de problemas de comportamento em crianças com idade entre 6 e 12 anos. Os resultados mostraram que meninos filhos de alcoolistas apresentaram mais agressão física, oposicionismo e hiperatividade, e mais ansiedade do que filhos de nãoalcoolistas $^{(22)}$. Os autores afirmaram que problemas de comportamento em filhos de alcoolistas surgem em idade mais precoce e tendem a persistir por mais tempo, tanto para meninas quanto para meninos.

Estudos demonstraram que meninos tendem a ser mais vulneráveis ao alcoolismo parental que as meninas, apresentando problemas comportamentais, afetivos e escolares. Pode-se atribuir tais resultados negativos ao comprometimento da figura paterna como modelo para os meninos. Entretanto, há crianças resistentes a esse fator de stress familiar, o que foi também verificado na investigação de meninos filhos de alcoolistas sem problemas de comportamento e meninos filhos de alcoolistas, que apresentavam problemas de comportamento (relatado pelos professores) e filhos de não-alcoolistas, com idades de 6 a 14 anos. Do acompanhamento dos três grupos, emergiram os seguintes resultados: filhos de \begin{tabular}{llll}
\hline SMAD 2005 & $\begin{array}{l}\text { Volume } \\
\text { Volumen }\end{array}$ & $\begin{array}{l}\text { Número } \\
\text { Numero } \\
\text { Number }\end{array}$ & $\begin{array}{l}\text { Artigo } \\
\text { Artículo } \\
\text { Article }\end{array}$
\end{tabular} 06 http://www2.eerp.usp.br/resmad/artigos.asp 
alcoolistas sem problemas de comportamento e filhos de não-alcoolistas apresentaram melhor desempenho escolar e menos problemas como delinqüência, abuso de substâncias e problemas escolares $^{(23)}$.

- Quanto ao uso/abuso de álcool e drogas

A literatura vem apontando que, dentre os resultados negativos que o alcoolismo parental pode ter, no desenvolvimento da criança e adolescente, o prognóstico de uso ou abuso de álcool e outras substâncias psicoativas é, talvez, o mais evidenciado. Através de estudo longitudinal, compararando crianças e adolescentes, vindos de famílias com alto e baixo risco para desenvolver alcoolismo e famílias de baixo risco para alcoolismo, verificouse que, crianças de famílias com alto risco começam a beber em idade mais precoce, sendo que a idade do início de beber tem sido mostrada em estudos da população geral como preditor de provável desenvolvimento de problemas de uso, abuso e dependência de álcool. Além disso, crianças de famílias de alto risco, quando desenvolveram abuso e dependência de álcool, o fizeram em níveis de maior gravidade quando comparadas a crianças de famílias de baixo risco. O estudo também demonstrou a existência de ligação entre preditores na infância, tais como temperamento extrovertido e desenvolvimento de problemas com álcool ${ }^{(24)}$.

Com o objetivo de examinar a influência e/ou a relação entre o status de ser filho de alcoolista e: auto-estima, idade, gênero e o uso de álcool, em 649 adolescentes (idade entre 12 e 19 anos), verificou-se inter-relação entre o status de ser filho de alcoolista e o abuso de álcool pelos mesmos, sendo que dos $52 \%$ dos adolescentes que se identificaram como abusadores de álcool, um em cada quatro era filho de alcoolista. Idade, baixa auto-estima e status de ser filho de alcoolista foram identificados como preditores do modo de beber entre estes jovens $^{(25)}$.

Outro fator apontado por pesquisadores na área do alcoolismo são as expectativas que filhos de alcoolistas têm em relação ao uso de álcool. Estudo examinando filhos de alcoolistas

$\begin{array}{llll}\text { SMAD } 2005 & \begin{array}{l}\text { Volume } \\ \text { Volumen }\end{array} & \begin{array}{l}\text { Número } \\ \text { Numero } \\ \text { Number }\end{array} & \begin{array}{l}\text { Artigo } \\ \text { Artículo } \\ \text { Article }\end{array}\end{array} \quad$ http://www2.eerp.usp.br/resmad/artigos.asp


e filhos de não-alcoolistas com idade entre 7 e 12 anos tinha como hipóteses que filhos de alcoolistas, poderiam apresentar expectativas mais negativas do que filhos de não-alcoolistas devido ao aprendizado ‘adverso’; ou expectativas mais positivas devido ao aprendizado social precoce e à exposição a respostas mais favoráveis ao álcool. Os resultados demonstraram que filhos de alcoolistas, em idade escolar, têm expectativas mais negativas em relação ao álcool que filhos de não-alcoolistas. Entretanto, os adolescentes filhos de alcoolistas têm expectativas mais positivas, e essas se associam a experiências próprias com o álcool. As expectativas em relação ao uso de álcool podem ser um importante inibidor ou estimulador para o uso do mesmo e os resultados do estudo sugerem evidências em favor dessa hipótese ${ }^{(26)}$.

Outro fator que vem se somar às expectativas de filhos de alcoolistas é o próprio funcionamento da família, que pode configurar-se em padrões disfuncionais. Além do uso do álcool poder estar associado, em parte, a um processo de socialização que valoriza o uso de bebida alcoólica, o abuso pode associar-se a disfunções familiares ${ }^{(27-28)}$.

O exame de comportamentos e concepções em relação ao uso de álcool em 270 jovens, com idade entre 16 e 18 anos, com ou sem história familiar de abuso de álcool, demonstrou que jovens com história familiar positiva de abuso de álcool tenderam a subestimar seus níveis de alcoolização quando comparados a indivíduos sem história familiar. Os achados sugerem que os julgamentos errados tendem a ser importante variável no processo das relações entre história familiar de alcoolismo e tendências de comportamentos relacionados com álcool ${ }^{(29)}$.

O que os estudos citados acima sugerem é que algumas crianças e adolescentes, mas não todas, sofrem conseqüências negativas devido ao alcoolismo parental. Outro estudo demonstrou que filhos de alcoolistas que não desenvolveram problemas com uso de álcool e outras drogas na adolescência são resilientes. Os autores focalizaram como fatores de

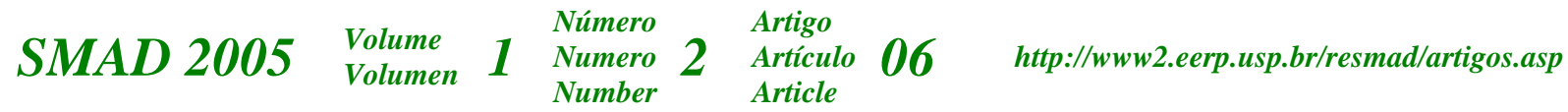


resiliência características da personalidade e do funcionamento familiar de crianças com idade entre 6 e 12 anos. A presença e a ausência de problemas com álcool e outras drogas foram avaliados posteriormente aos 15 e 16 anos de idade. Existia expectativa de que o alcoolismo parental seria importante fator de risco para o desenvolvimento de problemas com uso e abuso de substâncias nos adolescentes. O risco, entretanto, diminuiu quando foram identificadas características pessoais como tendência à inibição e baixo nível de buscas de emoções fortes e supervisão parental $^{(30)}$. Esses seriam, portanto, fatores de proteção à criança e adolescente.

Disfunções familiares são fatores que, associados ao alcoolismo parental, criam condições de vulnerabilidade para crianças e adolescentes, porém, características pessoais podem se constituir em fatores de proteção ou fatores de risco para o uso de álcool.

- Aspectos interacionais - pai-filhos e criança-criança

Embora uma série de fatores contribua para a etiologia do alcoolismo, vários estudos têm examinado os comportamentos e interações entre pais e filhos, e a análise de seus resultados leva a uma forte ligação entre processos familiares e desempenho dos filhos. Em um estudo, crianças com idade de 3 a 6 anos foram avaliadas durante uma sessão de ludoterapia. As crianças foram selecionadas a partir do risco de desenvolver alcoolismo, sendo crianças de alto risco e crianças de baixo risco. Crianças filhas de alcoolistas exibiram mais inibição de comportamento, levando significantemente mais tempo para começar as atividades e interagindo menos com as outras crianças $^{(31)}$.

Com relação às interações de crianças e adolescentes com seus pais e a avaliação da qualidade dessas interações com o alcoolismo parental evidenciou-se que o alcoolismo dos pais foi associado com mais interações negativas e indicou baixa sensibilidade e afeto negativo, menos verbalizações e baixo nível de responsividade à criança. Os resultados sugerem que os riscos para posterior mau ajustamento entre filhos de alcoolistas aparentemente surgem precocemente na infância ${ }^{(32)}$. Em estudo similar, verificaram-se fortes \begin{tabular}{lllll}
\hline SMAD 2005 & $\begin{array}{l}\text { Volume } \\
\text { Volumen }\end{array}$ & $\begin{array}{l}\text { Número } \\
\text { Numero } \\
\text { Number }\end{array}$ & $\begin{array}{l}\text { Artigo } \\
\text { Artículo } \\
\text { Article }\end{array}$ & 06
\end{tabular} http://www2.eerp.usp.br/resmad/artigos.asp 
relações entre problemas de comportamento nos filhos e dificuldades no relacionamento familiar, sendo que isso aparece mais intensamente em famílias em que o casal ou apenas a mãe era alcoolista ${ }^{(33)}$.

Testando a hipótese de que o suporte parental modera os efeitos dos conflitos entre pais e adolescentes e problemas de comportamento em adolescentes, examinando fatores de risco familiar, alcoolismo parental e conflitos entre pais e adolescentes, os resultados mostraram que conflitos familiares estavam altamente relacionados a problemas de comportamento quando o suporte parental era baixo. Conflitos entre pais e adolescentes foram relacionados com problemas de comportamento para filhos de alcoolistas, mas não para filhos de não-alcoolistas ${ }^{(34)}$.

Conflitos de relacionamento pais-crianças e características afetivas negativas dos pais, em co-ocorrência com o alcoolismo parental, são os elementos que tornam a criança e/ou adolescente vulnerável.

- Quanto a respostas perceptivas (ou de atenção)

Estudos de alto risco para o desenvolvimento de alcoolismo têm, comumente, abordado sujeitos que apresentam história de alcoolismo multigeracional. A comparação entre crianças em idade escolar e pré-adolescente, com famílias com múltiplas gerações de alcoolistas e crianças com um pai alcoolista ou sem pais alcoolistas mostrou diferenças significativas em seus desempenhos apenas para crianças com alcoolismo multigeracional. Crianças com alcoolismo multigeracional apresentam resultados inferiores em tarefas de discriminação visoespacial ${ }^{(35-38)}$.

Tais estudos sugerem que déficits de atenção podem estar associados ao alcoolismo parental e atuam como fator de vulnerabilidade para a criança. O mecanismo de instalação do déficit ocorreria através da hereditariedade. Entretanto, os estudos não são unânimes em confirmar a presença de déficits em filhos de alcoolistas.

\begin{tabular}{llll}
\hline SMAD 2005 & $\begin{array}{l}\text { Volume } \\
\text { Volumen } 1\end{array}$ & $\begin{array}{l}\text { Número } \\
\text { Numero } \\
\text { Number }\end{array}$ & $\begin{array}{l}\text { Artigo } \\
\text { Artículo } 06 \\
\text { Article }\end{array}$
\end{tabular} http://www2.eerp.usp.br/resmad/artigos.asp




\section{2) Co-ocorrência de alcoolismo com outro distúrbio e características da criança ou do}

\section{adolescente}

- Comportamento anti-social dos pais

O alcoolismo comumente pode estar associado a comportamento anti-social. Examinando famílias com pais alcoolistas, que também exibiam comportamentos anti-sociais, famílias cujos pais eram apenas alcoolistas e famílias de não-alcoolistas os pesquisadores verificaram que filhos com pais alcoolistas e problemas comportamentais também exibiam mais problemas de comportamento externalizados ou internalizados. Meninos foram mais afetados que meninas. Crianças em idade escolar exibiam problemas de comportamento do tipo internalizado ${ }^{(39)}$. Outros pesquisadores mostraram a associação entre alcoolismo e comportamento anti-social dos pais e sua influência deletéria sobre o desenvolvimento das $\operatorname{crianças~}^{(40)}$.

- Eventos adversos na família

Eventos estressores do ambiente familiar, comum em famílias de alcoolistas, podem contribuir para o risco dos problemas observados em filhos de alcoolistas. Em investigação sobre a existência de relações entre ambiente familiar e experimentação de álcool e outras substâncias psicoativas os mais fortes preditores da experimentação precoce de álcool e outras substâncias foram eventos e experiências negativas na vida, conflitos familiares e falta de coesão familiar, em contraposição a ser apenas filho de alcoolista. Diferenças foram achadas entre filhos de alcoolistas e filhos de não-alcoolistas com relação à estrutura familiar, nível educacional dos pais e ambiente familiar. Filhos de alcoolistas mostraram ter mais probabilidade, que filhos de não-alcoolistas, de experimentar tabaco, mas não álcool e $\operatorname{drogas}^{(41)}$.

\section{3) Fatores que podem funcionar como protetores à criança/adolescente}


Existem alguns fatores que podem funcionar como protetores à saúde mental das crianças e dos adolescentes que vivem com o alcoolismo parental, desde que exista a ausência de sintomas de comprometimento psicológico e quando a família é competente em prover as necessidades materiais e afetivas. Existem fortes correlações entre a competência familiar e o status socioeconômico ${ }^{(42)}$. Altos níveis de organização familiar e comportamento de coping podem deter ou diminuir a iniciação do jovem no uso de substâncias. Além disso, se o adolescente percebe sua família como organizada, ele próprio sente que tem controle pessoal $^{(43)}$.

Quando o membro não-alcoolista é responsável e preocupado em manter a rotina familiar, e quando a criança ou o adolescente consegue manter um bom relacionamento com o membro não-alcoolista e/ou com irmãos, o impacto do alcoolismo parental tende a $\operatorname{diminuir}^{(44)}$.

\section{CONCLUSÕES}

Dos resultados obtidos através da revisão bibliográfica, observa-se que apenas um trabalho publicado em periódico nacional, mas realizado no exterior, que aborda adultos filhos de alcoolistas, sendo todos os outros publicações internacionais. O que, em parte, demonstra a inexistência de pesquisas que abordem crianças e adolescentes e as repercussões do alcoolismo parental no desenvolvimento dos filhos.

Através da análise desses estudos, constatou-se que, atualmente, existe tendência em reconhecer que o alcoolismo não afeta somente a pessoa alcoolista, pois, além do comprometimento físico e mental do indivíduo, a doença repercute nos familiares que convivem mais diretamente com a pessoa alcoolista. 
Outro ponto que a literatura vem apontando é a freqüência com que o alcoolismo tende a recorrer nas famílias, fato que, isoladamente, justificaria o aprofundamento de estudos sobre essa temática. Em nosso país, mesmo diante do número de pessoas afetadas pelo alcoolismo, encontramos poucos estudos que focalizam na mesma proporção a pessoa alcoolista e o seu contexto sóciofamiliar. Diante do exposto, fica evidente a necessidade de atentar-se para aspectos do funcionamento psicológico das crianças e adolescentes filhos de pais alcoolistas em nosso meio. 


\section{REFERÊNCIAS BIBLIOGRÁFICAS}

1- Almeida LM, Coutinho ESF. O alcoolismo e o hospital geral. Estudo de prevalência junto à demanda ambulatorial. J Bras Psiquiatr 1990; 39 (1): 27-31.

2- Almeida LM. Coutinho ESF. Prevalência de consumo de bebidas alcoólicas e de alcoolismo em uma região metropolitana do Brasil. Rev Saúde Pública 1993; 27 (1): 23-39.

3- Bucher R. Drogas: O que é preciso Saber. São Paulo (SP): Governo do Estado de São Paulo, 1992.

4- Grant BF. Estimates of US children exposed to alcohol abuse and dependence in the family. Am J Public Health 2000; 90 (1): 112-15.

5- Windle M. Concepts and issues in COA research. Alcohol Res Health 1997; 21 (3): 185-191.

6- Jacob T, Leonard K. Psychological functioning in children of alcoholic fathers, depressed fathers and control fathers. J Stud Alcohol 1986; 47: 343-80.

7- Jacob T, Johnson S. Parenting Influences on the Development of Alcohol Abuse and Dependence. Alcohol Res Health 1997; 21 (3): 204-9.

8- Windle M, Searles JS. Children of Alcoholics: Critical Perspectives. New York: Guilford Press; 1990.

9- Clair D, Genest M. Variables associated with the adjustment of offspring of alcoholic fathers. J Stud Alcohol 1986; 48 (4): 345-55.

10- Moss RH, Billings AG. Children of alcoholics during the recovery process: Alcoholic and matched control families. Addict Behav 1982; 7: 155-63.

11- National Institute on Alcohol Abuse And Alcoholism. Children of Alcoholics: Are They Different? July 1990; 9 (RR288).

12- Chassin L, Rogosch F, Barrera M. Substance use and symptomatology among adolescent children of alcoholics. J Abnorm Psychol 1991; 100: 449-63.

13- Pandina RJ, Johson V. Serious alcohol and drug problems among adolescents with a family history of alcoholism. J Stud Alcohol 1990; 51: 278-82.

14- Russell M. Prevalence of alcoholism among chidren of alcoholics. In: Windle M, Searles JS, editors. Children of Alcoholics: Critical Perspectives. New York: Guilford Press; 1990. p. 9-38. 15- West MO, Prinz RJ. Parental alcoholism and childhood psychopathology. Psychol Bull 1987; 102: 204-218.

16- Seilhamer RA, Jacob T. A Family factors and adjustment of children of alcoholics. In: Windle M, Searles JS editors. Children of Alcoholics: Critical Perspectives. New York: Guilford Press; 1990. p. 168-86.

17- Bronfenbrenner U. The ecology of human development: experiments by nature and design. Cambridge, Mass: Harvard University Press; 1979.

18- Mcgrath CE, Watson AL, Chassin L. Academic achievement in adolescent children of alcoholics. J Stud Alcohol 1999; 60 (1): 18-26.

19- Wall T, Garcia-Andrade C, Wong V, Lau P, Ehlers CL. Parental history of alcoholism and problem behaviors in native-american children and adolescents. Alcohol Clin Exp Res 2000; 24: (1): 30-4.

20- Hill SY, Locke J, Lowers L, Connolly J. Psychopathology and achievement in children at high risk for developing alcoholism. J Am Acad Child Adolesc Psychiatry 1999; 38 (7): 883-91.

21- Morey CK. Children of alcoholics: a school-based comparative study. J Drug Educ 1999; 29 (1): 63-75.

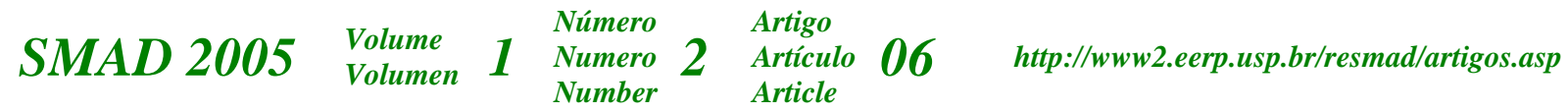


22- Carbonneau R, Tremblay RE, Vitaro F, Dobkin PL, Saucier JF, Pihl RO. Paternal alcoholism, paternal absence and the development of problem behaviors in boys from age six to twelve years. J Stud Alcohol 1998; 59: 387-98.

23- Vitaro F, Dobkin PL, Carbonneau R, Tremblay RE. Personal and familial characteristics of resilient sons of male alcoholics. Addiction 1996; 91 (8): 1161-77.

24- Hill SY, Yuan H. Familial density of alcoholism and onset of adolescent drinking. J Stud Alcohol 1999; 60 (1): 7-17.

25- Rodney HE, Mupier R, Crafter B. Predictors of alcohol drinking among African American adolescents: Implications for violence prevention. J Negro Educ 1996; 65 (4): 434-44. 26- Wiers RW, Gunning WB, Sergeant JA. Is a mild deficit in executive functions in boys related to childhood ADHD or to parental multigenerational. J Abnorm Child Psychol 1998; 26 (6): 415-30.

27- Colder CR, Chassin L. The psychosocial characteristics of alcohol users versus problem users: Data from a study of adolescents at risk. Dev Psychopathol 1999; 11 (2): 321-48.

28- Brown SA, Tate SR, Vik PW, Haas AL, Aarons GA. Modeling of alcohol use mediates the of family history of alcoholism on adolescent alcohol expectancies. Exp Clin Psychopharmacol 1999; 7 (1): 20-7.

29- Turrisi R, Wiersma K. Examination of judgments of drunkenness, binge drinking, and drunk-driving tendencies in teens with and a family history of alcohol abuse. Alcohol Clin Exp Res 1999; 23 (7): 1191-98.

30- Vitaro F, Tremblay RE, Zoccolillo M. Alcoholic father, adolescent drug abuse and protective factores. Can J Psychiatry 1999; 44 (9): 901-8.

31- Hill SY, Lowers L, Locke J, Sinidman N, Kagan J. Behavioral inhibition in children from families at high risk for developing alcoholism. J Am Acad Child Adolesc Psychiatry 1999; 38 (4): 410-20.

32- Eiden RD, Chavez F, Leonard KE. Parent-infant interactions among families with alcoholic fathers. Dev Psychopathol 1999; 11 (4): 745-62.

33- Moser RP, Jacob T. Parent-child interactions and child as related to gender of alcoholic parent. J Subst. Abuse Treat 1997; 9: 189-208.

34- Barrera MJR, Stice E. Parent-adolescent conflict in the context of parental support: Families with alcoholic and nonalcoholic fathers. J Fam Psychol 1998; 12 (2): 195-208.

35- Corral MM, Holguin SR, Cadaveira F. Neuropsychological characteristics in children of alcoholics: familial density. J Stud Alcohol 1999; 60 (4): 509-13.

36- Holguin RS, Corral M, Cadaveira F. Event-related potentials elicited by infrequent nontarget stimuli in Young children of alcoholics: family history and gender differences. Alcohol Alcohol 1998; 33 (3): 281-90.

37- Holguin SR, Corral M, Cadaveira F. Mismatch negativity in Young children of alcoholics from high-density families. Alcohol Clin Exp Res 1998; 22 (6): 1363-8.

38- Schandler SL, Thomas CS, Cohen MJ. Spatial learning deficits in preschool children of alcoholics. Alcohol Clin Exp Res 1995; 19 (4): 1067-72.

39- Puttler LI, Zucker RA, Fitzgerald HE, Bingham CR. Behavioral outcomes among children of alcoholics during the early and middle childhood years: familial subtype variations. Alcohol Clin Exp Res 1998; 22 (9): 1962-72.

40- Wong MM, Zucker RA, Puttler LI, Fitzgerald HE. Heterogeneity of risk aggregation for alcohol problems between early and middle childhood: nesting structure variations. Dev. Psychopathol 1999; 11 (4): 727-44.

41- Havey JM, Dodd DK. (1995). Children of alcoholic, negative life, and early experimentation with drugs. J School Psychol 1995; 33 (4): 305-17.

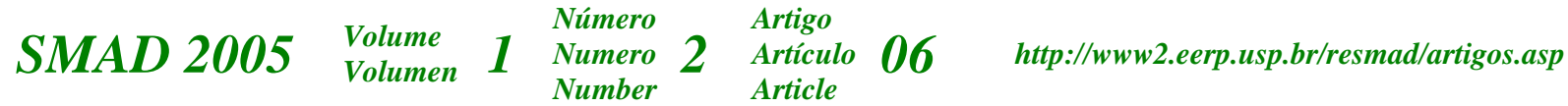


42- Haugland BS, Havik OE. Correlates of family competence in families with paternal alcohol abuse. Psychol Rep 1998; 83 (3): 867-80.

43- Hussong AM, Chassin L. Substance use initiation among adolescent children of alcoholics: Testing protective factors. J Stud Alcohol 1997; 58 (3): 272-79.

44- Walker JP, Lee RE. Uncovering strengths of children of alcoholic parents. Contemp Fam Ther 1998; 20 (4): 521-38.

$(4)$
$(5)$
$(6)$
$(7,8,9,10)$
$(11)$
$(12,13,14)$
$(17)$
$(18)$
$(19)$
$(20)$
$(21)$
$(22)$
$(23)$
$(24)$
$(25)$
$(26)$
$(29)$
$(30)$
$(31)$
$(32)$
$(33)$
$(34)$
$(35-38)$
$(39)$
$(40)$
$(41)$
$(42)$
$(43)$
$(44)$

